\title{
16 Unart aus therapeutischer Sicht
}

Kreative Prozesse können einen wesentlichen Beitrag zur Identitätsbildung leisten und zu einer Stärkung des Selbstvertrauens führen. Deshalb nehmen die in der Prof. Eggers-Stiftung betreuten jungen Menschen an einem speziellen Kunstprojekt teil, welches wir „Un-Art“ genannt haben. Dieser Begriff weckt Assoziationen: UnArt = Nicht-Kunst. Die Frage stellt sich: was ist Kunst? Und: hat das Projekt etwas mit Kunst zu tun? Diese Frage ist in unserem Zusammenhang nicht so wichtig und wir halten uns pragmatisch an das berühmt gewordene Diktum von Joseph Beuys: „Jeder Mensch ist ein Künstler“.

Der Begriff Un-Art hat aber auch etwas mit un-artig zu tun. Was bedeutet es, unartig zu sein? Es hat etwas mit der Entdeckung des eigenen Willens zu tun. Das ist gleichzusetzen mit der Entdeckung des eigenen, wahren, lebendigen Wesens, welches dieses Selbst repräsentiert, Authentizität, Eigenständigkeit, Individualität und Autonomie. Es macht das eigentliche Wesen des Menschen aus.

In der Kunst spiegelt sich etwas vom eigentlichen Wesen des Menschen wider, hier entdeckt er sich, hier tritt er in eine gemütshafte Beziehung zu sich selbst. Der Mensch drückt sich im künstlerischen Gestalten aus, aber er erkennt sich auch im Kunstwerk wieder. Dieses Doppel-Verhältnis des Menschen zur Kunst gehört somit zum Wesen der Kunst. In der Kunst entdeckt der gestaltende Mensch ebenso wie der betrachtende Mensch sein eigenes Wesen. Sein Wesen entdecken, d.h. letztlich, der Mensch setzt sich als ein sich aus-setzendes Wesen der Wahrheit aus. Heidegger würde sagen: Der Wahrheit des Seins.

Von Sigmund Freud stammt der Satz, dass Kunst nichts anderes sei als gescheiterte soziale Anpassung. In der Tat, der angepasste Mensch verfügt im Grunde nur über eine Leih-Identität, für die äußere Normen das Gerippe darstellen, er ist also nur ein „Pseudo-Selbst“. Er mag zwar artig sein, vermag aber nicht aus sich selbst heraus zu existieren. Kunst wird von ihm nicht selten als fremd und unverständlich empfun- 
den. Denn Kunst ist, so hat es Hegel gesehen, ein Phänomen des menschlichen Daseins, das vom objektiv erkennenden Geist überwunden werden muss, damit die Idee in der „unendlichen Freiheit des begreifenden Denkens“ rein aufleuchten kann.

Hier besteht also eine wesentliche Übereinstimmung im Denken von Friedrich Wilhelm Hegel und Sigmund Freud sowie von D.W. Winnicott, wie aus dem umseitigen Zitat ersichtlich ist.

Auch der sogenannte psychisch Kranke, das Kind, der Jugendliche, dessen gefühlshafte und lebendige Entwicklung durch widrige Umstände beeinträchtigt ist, weshalb er unsere Hilfe in Anspruch nimmt, begegnet im künstlerischen Gestalten seinem eigenen Wesen. Das Sich-Ausdrücken im Bildhaften ist auch für ihn die Möglichkeit, einen Zugang zu gewinnen zu seinen sonst verborgenen Konflikten, Ängsten und Problemen. In der originalen Leistung des bildnerischen Gestaltens spiegeln sich dann auch verborgene Ich-Kräfte wider, die durch den kreativen Prozess des Malens und Gestaltens zum Vorschein kommen und wachsen können.

Im bildnerischen oder modellierenden Gestalten kann sich das wahre, lebendige Selbst, der tiefe, gemüthafte Kern des Kranken entwickeln. Die verschiedenen seelischen Krankheitssymptome sind letztlich Ausdruck einer gestörten Entwicklung in der Kindheit des Patienten, der als Kind in seinem eigentlichen Sein von seiner engsten Umgebung, häufig von den Eltern, nicht wahrgenommen werden konnte, teilweise sogar entwertet und verneint wurde: ein teufelskreisartiger Prozess, der damit zusammenhängt, dass die Eltern wiederum von ihren eigenen Eltern in ihrem eigentlichen Wesen nicht haben wahrgenommen werden können.

Eben weil es um seelische Entwicklung geht, sprechen wir nicht von „psychischer Krankheit“, sondern vielmehr von Entwicklungsstörungen. Wir meinen mit Winnicott, dass seelische Gesundheit eine Frage der Reife, nicht aber eine Frage des Freiseins von Symptomen ist. Bei allem psychischen Erkrankungen geht es letztlich um eine gestörte seelische Entwicklung des betroffenen Individuums, dem im therapeutischen Prozess dazu verholfen werden muss, fehlende Entwicklungsschritte nachzuholen und sich zu einer lebendigen, autonomen Persönlichkeit hin zu entwickeln bzw. ihm, was dasselbe ist, die Entfaltung seines wahren lebendigen Selbst zu ermöglichen. Es geht also nicht um das Kurieren von Krankheitssymptomen, sondern um den Anstoß schöpferischer Prozesse; insofern muss der traditionelle Krankheitsbegriff im Bereich der Psychiatrie neu reflektiert werden.

Indem ich den Patienten zum „Objekt“ einer Behandlung mache, ohne wirklich mit ihm in eine persönliche, sich den Problemen und Konflikten des Patienten gemeinsam aus-setzende Beziehung zu kommen, reduziere ich ihn. Diese Reduzierung kann soweit gehen, dass der Patient dann als „defekt“ oder sonst wie abnorm gekennzeichnet wird. Damit tritt aber eben gerade die gleiche Entwertung ein, die der Patient schon von jeher erfahren hat, und es bleibt ihm auch nichts anderes übrig, als sich gemäß dem Prinzip der „self-fullfilling prophecy“ entsprechend den Erwartungen und Zuschreibungen seiner Umgebung zu verhalten, was jeden Entwicklungsprozess verunmöglicht.

Ganz im Gegenteil geht es bei dem gemeinsamen, kreativen, unprogrammierten Gestalten, Malen, Modellieren mit den Patienten um die gemeinsame Entwicklung aller Beteiligten, so wie es in einer guten Therapie immer auch nur um die gemeinsame Entwicklung von Therapeut und Patient geht. Das eine ist vom anderen abhän- 
gig, die Entwicklung des Patienten ist nur soweit möglich, als sich auch der Therapeut in der Beziehung entwickeln kann.

In dieser Beziehung kommt es darauf an, dass dem Patienten auch gestattet wird, auf je seine eigene Weise „verrückt" zu sein, eine „Verrücktheit“, die man sonst nur Kleinkindern zugesteht. Diese Phase ist eine wichtige Entwicklungsphase im Übergang vom magisch-animistischen Welterleben des Kleinkindes zur rational-kritischen Reflexionsfähigkeit des älteren Schulkindes, des Jugendlichen und schließlich des Erwachsenen.

Im gemeinsamen künstlerischen Tun mit unseren Patienten, sowie wir es verstehen, kann eine „genügend gute Umwelt“ (Winnicott) geschaffen werden, die es dem Patienten gestattet, seine in der Kindheit unausgelebte „Verrücktheit“ nachzuholen und an ihr zu reifen.

Nur indem sich im gemeinsamen kreativen Tun Subjekt mit Subjekt begegnet, ist Reifung im Sinne einer Entwicklung des wahren, lebendigen Selbst möglich. So entsteht das Paradoxon, dass ein Geschehen, das primär und ursprünglich eben keine Therapie, also auch keine Kunsttherapie sein soll, in ganz besonderem Maße therapeutisch wirksam ist. 